\title{
Upper Semicontinuity of Solution Mappings to Parametric Generalized Vector Quasiequilibrium Problems
}

\author{
Shu-qiang Shan, Yu Han, and Nan-jing Huang \\ Department of Mathematics, Sichuan University, Chengdu, Sichuan 610064, China \\ Correspondence should be addressed to Nan-jing Huang; nanjinghuang@hotmail.com
}

Received 11 December 2014; Revised 16 March 2015; Accepted 25 March 2015

Academic Editor: Mohamed-Aziz Taoudi

Copyright (c) 2015 Shu-qiang Shan et al. This is an open access article distributed under the Creative Commons Attribution License, which permits unrestricted use, distribution, and reproduction in any medium, provided the original work is properly cited.

\begin{abstract}
We establish the upper semicontinuity of solution mappings for a class of parametric generalized vector quasiequilibrium problems. As applications, we obtain the upper semicontinuity of solution mappings to several problems, such as parametric optimization problem, parametric saddle point problem, parametric Nash equilibria problem, parametric variational inequality, and parametric equilibrium problem.
\end{abstract}

\section{Introduction}

It is well known that the vector equilibrium problem provides a unified model of several problems, such as the vector optimization problem, the vector saddle point problem, the vector complementarity problem, and the vector variational inequality problem $[1,2]$. In recent years, the existence of solutions for various types of vector equilibrium problems has been investigated intensively by many authors under different conditions (see, e.g., [3-8] and the references therein).

On the other hand, the stability analysis of the solution mapping to vector equilibrium problems is an important topic in vector optimization theory. In recent years, the lower semicontinuity and the upper semicontinuity of of the solution mappings to parametric optimization problems, parametric vector variational inequalities, and parametric vector equilibrium problems have been intensively studied in the literature; for instance, we refer the reader to [9-17]. Recently, Anh and Khanh [18] obtained the semicontinuity of the solution mapping to parametric vector quasiequilibrium problems. Khanh and Luu [19] discussed the upper semicontinuity of solution mapping to parametric vector quasivariational inequalities involving multifunctions without monotonicity assumptions. Fang and Huang [20] established upper semicontinuity of the solution maps to the vector homogeneous quasiequilibrium problems. By using a scalarization method, Cheng and Zhu [21] investigated the upper semicontinuity and lower semicontinuity of the solution mapping to a parametric weak vector variational inequality in finite-dimension Euclidean spaces. Li and Fang [22] studied the lower semicontinuity of the solution mappings to a parametric generalized Ky Fan inequality by using a key assumption that includes the information about the solutions set. By virtue of a density result and scalarization technique, Gong and Yao [23] first discussed the lower semicontinuity of the set of efficient solutions to parametric vector equilibrium problems. Li et al. [24] investigated the upper semicontinuity and lower semicontinuity of the solution mappings to a parametric generalized strong vector equilibrium problem. By using the Hölder relation, Zhang et al. [25] obtained the lower semicontinuity of the efficient solution mapping to a parametric vector equilibrium problem. Fan et al. [26] studied the continuity of the solution mapping concerned with a class of vector quasiequilibrium problems with an application to traffic network problems. $\mathrm{Xu}$ and $\mathrm{Li}$ [27] established the lower semicontinuity of solution mappings to a parametric generalized strong vector equilibrium problem by using a scalarization method. Very recently, by using a new proof method which is different from the ones used in the literature, Han and Gong [28] established the lower semicontinuity of the solution mappings to parametric generalized strong vector equilibrium problems without the assumptions of monotonicity and compactness. 
The aim of this paper is to establish the upper semicontinuity of solution mappings for a class of parametric generalized vector quasiequilibrium problems under some suitable conditions. We provide a uniform method to deal with the upper semicontinuity of solution mappings for several problems, such as parametric optimization problem, parametric saddle point problem, parametric Nash equilibria problem, parametric variational inequality, and parametric equilibrium problem. The rest of the paper is organized as follows. In Section 2, we recall some basic concepts and known lemmas. In Section 3, we show a main result in connection with the upper semicontinuity of the solution mapping for the parametric generalized vector quasiequilibrium problem. Some applications of the main result are given in Section 4.

\section{Preliminaries}

Throughout this paper, unless otherwise specified, let $\Lambda, W$, $X$, and $Y$ be four normed vector spaces. Let $D$ be a nonempty closed subset of $Y$. Let $F: X \times X \times W \rightarrow 2^{Y}$ and $K$ : $\Lambda \rightarrow 2^{X}$ be two set-valued mappings. For $(u, \lambda) \in W \times \Lambda$, we consider that the following parametric generalized vector quasiequilibrium problems consist of finding $x_{0} \in K(\lambda)$ such that

$$
F\left(x_{0}, y, u\right) \subseteq D, \quad \forall y \in K(\lambda)
$$

(PGVQEP)

We define a solution mapping $S$ to (PGVQEP) by

$$
\begin{array}{r}
S(u, \lambda)=\{x \in K(\lambda): F(x, y, u) \subseteq D, \quad \forall y \in K(\lambda)\} \\
(u, \lambda) \in W \times \Lambda .
\end{array}
$$

Definition 1 (see [29]). Let $T$ and $Q$ be two topological vector spaces. A set-valued mapping $G: T \rightarrow 2^{\mathrm{Q}}$ is said to be

(i) upper semicontinuous (u.s.c.) at $u_{0} \in T$ if, for any neighborhood $V$ of $G\left(u_{0}\right)$, there exists a neighborhood $U\left(u_{0}\right)$ of $u_{0}$ such that, for every $u \in U\left(u_{0}\right)$, $G(u) \subseteq V$;

(ii) lower semicontinuous (l.s.c.) at $u_{0} \in T$ if, for any $x \in G\left(u_{0}\right)$ and any neighborhood $V$ of $x$, there exists a neighborhood $U\left(u_{0}\right)$ of $u_{0}$ such that, for every $u \in$ $U\left(u_{0}\right), G(u) \cap V \neq \emptyset$.

A set-valued mapping $G$ is said to be u.s.c. and l.s.c. on $T$, if it is u.s.c. and l.s.c. at each $u \in T$, respectively. We say that $G$ is continuous on $T$, if it is both u.s.c and l.s.c on $T$.

Definition 2 (see [15]). Let $T$ and $Q$ be two topological vector spaces and let $C \subseteq Q$ be a cone. A set-valued mapping $G$ : $T \rightarrow 2^{\mathrm{Q}}$ is said to be $C$-lower semicontinuous (C-l.s.c.) at $u_{0} \in T$ if, for any $x \in G\left(u_{0}\right)$ and any neighborhood $V$ of $x$, there exists a neighborhood $U\left(u_{0}\right)$ of $u_{0}$ such that

$$
G(u) \cap(V-C) \neq \emptyset, \quad \forall u \in U\left(u_{0}\right) .
$$

A set-valued mapping $G$ is said to be $C$-l.s.c. on $T$, if it is $C$ l.s.c. at each $u \in T$.
Remark 3. It is easy to see that if $G$ is l.s.c. at $u_{0} \in T$, then it is $C$-l.s.c. at $u_{0} \in T$. In fact, since $0 \in C$, we have $V \subseteq V-C$. It follows from $G(u) \cap V \neq \emptyset$ that

$$
G(u) \cap(V-C) \neq \emptyset .
$$

The following example shows that the reverse is not true in general.

Example 4. Let $C=\mathbb{R}_{+}=\{x \in \mathbb{R}: x \geq 0\}$. We define a set-valued mapping $G: \mathbb{R} \rightarrow 2^{\mathbb{R}}$ as follows:

$$
G(x)= \begin{cases}{[\cos x, \sin x+2],} & x \neq 0, \\ {[1,3],} & x=0 .\end{cases}
$$

It is easy to see that $G$ is $C$-l.s.c. at 0 , but $G$ is not l.s.c. at 0 .

Lemma 5 (see [29]). A set-valued mapping $G: T \rightarrow 2^{\mathrm{Q}}$ is l.s.c. at $u_{0} \in T$ if and only if, for any sequence $\left\{u_{n}\right\} \subseteq T$ with $u_{n} \rightarrow u_{0}$ and for any $x_{0} \in G\left(u_{0}\right)$, there exists $x_{n} \in G\left(u_{n}\right)$ such that $x_{n} \rightarrow x_{0}$.

Lemma 6 (see [30]). Assume that $G: T \rightarrow 2^{Q}$ is a setvalued mapping. If $G\left(u_{0}\right)$ is compact for some $u_{0} \in T$, then $G$ is u.s.c. at $u_{0} \in T$ if and only if, for any sequence $\left\{u_{n}\right\} \subseteq T$ with $u_{n} \rightarrow u_{0}$ and for any $x_{n} \in G\left(u_{n}\right)$, there exist $x_{0} \in G\left(u_{0}\right)$ and a subsequence $\left\{x_{n_{k}}\right\}$ of $\left\{x_{n}\right\}$ such that $x_{n_{k}} \rightarrow x_{0}$.

\section{Upper Semicontinuity of Solution Mapping to (PGVQEP)}

In this section, we establish the upper semicontinuity of $S$ at $\left(u_{0}, \lambda_{0}\right) \in W \times \Lambda$.

Theorem 7. Let $\left(u_{0}, \lambda_{0}\right) \in W \times \Lambda$. Assume that $K\left(\lambda_{0}\right)$ is nonempty compact, $K(\cdot)$ is continuous at $\lambda_{0}$, and $F(\cdot, \cdot, \cdot)$ is l.s.c. on $K\left(\lambda_{0}\right) \times K\left(\lambda_{0}\right) \times\left\{u_{0}\right\}$. Then $S$ is u.s.c. at $\left(u_{0}, \lambda_{0}\right)$.

Proof. Suppose to the contrary that $S(\cdot, \cdot)$ is not u.s.c. at $\left(u_{0}, \lambda_{0}\right)$. Then there exists a neighborhood $W_{0}$ of $S\left(u_{0}, \lambda_{0}\right)$; for any neighborhood $U\left(u_{0}\right) \times U\left(\lambda_{0}\right)$ of $\left(u_{0}, \lambda_{0}\right)$, there exists $\left(u^{\prime}, \lambda^{\prime}\right) \in U\left(u_{0}\right) \times U\left(\lambda_{0}\right)$ such that $S\left(u^{\prime}, \lambda^{\prime}\right) \not \subset W_{0}$. Hence, there exists a sequence $\left\{\left(u_{n}, \lambda_{n}\right)\right\}$ with $\left(u_{n}, \lambda_{n}\right) \rightarrow\left(u_{0}, \lambda_{0}\right)$ such that

$$
S\left(u_{n}, \lambda_{n}\right) \not \subset W_{0}, \quad \forall n \in \mathbb{N} \text {. }
$$

Then there exists

$$
x_{n} \in S\left(u_{n}, \lambda_{n}\right)
$$

such that

$$
x_{n} \notin W_{0}, \quad \forall n \in \mathbb{N} .
$$

Since $K(\cdot)$ is u.s.c. at $\lambda_{0}$ and $x_{n} \in K\left(\lambda_{n}\right)$, by Lemma 6 , there exist $x_{0} \in K\left(\lambda_{0}\right)$ and a subsequence $\left\{x_{n_{k}}\right\}$ of $\left\{x_{n}\right\}$ such that $x_{n_{k}} \rightarrow x_{0}$. Without loss of generality, we can assume that $x_{n} \rightarrow x_{0}$. 
We claim that $x_{0} \in S\left(u_{0}, \lambda_{0}\right)$. In fact, suppose that $x_{0} \notin$ $S\left(u_{0}, \lambda_{0}\right)$. Then there exists $y_{0} \in K\left(\lambda_{0}\right)$ such that

$$
F\left(x_{0}, y_{0}, u_{0}\right) \not \subset D \text {. }
$$

Hence there exists $z_{0} \in F\left(x_{0}, y_{0}, u_{0}\right)$ such that

$$
z_{0} \notin D \text {. }
$$

Since $K(\cdot)$ is l.s.c. at $\lambda_{0}$ and $y_{0} \in K\left(\lambda_{0}\right)$, by Lemma 5 , there exists $y_{n} \in K\left(\lambda_{n}\right)$ such that $y_{n} \rightarrow y_{0}$. Since $F(\cdot, \cdot, \cdot)$ is l.s.c. at $\left(x_{0}, y_{0}, u_{0}\right)$ and $z_{0} \in F\left(x_{0}, y_{0}, u_{0}\right)$, by Lemma 5 , there exists $z_{n} \in F\left(x_{n}, y_{n}, u_{n}\right)$ such that $z_{n} \rightarrow z_{0}$. Noting (9) and that $D$ is closed, we know that $z_{n} \notin D$ for $n$ large enough, which contradicts with (6). Therefore, $x_{0} \in S\left(u_{0}, \lambda_{0}\right)$. It is easy to see that $x_{n} \rightarrow x_{0} \in W_{0}$, which contradicts with (7).

Remark 8. We would like to point out that the assumptions of Theorem 7 are quite natural and easy to be verified.

We give an example to illustrate Theorem 7.

Example 9. Let $Y=\mathbb{R}^{2}, D=\left\{\left(x_{1}, x_{2}\right) \in \mathbb{R}^{2}: x_{1} \leq 0, x_{2} \geq\right.$ $0\}$, and $\Lambda=W=X=\mathbb{R}$. Let $B$ be the closed unit ball of $\mathbb{R}^{2}$. Assume that $F: X \times X \times W \rightarrow 2^{Y}$ is defined by

$$
F(x, y, u)=\left(f_{1}(x, y, u), f_{2}(x, y, u)\right)+B,
$$

where

$$
\begin{gathered}
f_{1}(x, y, u)=\ln x-x y-2^{y}-y^{2} \cos u \\
+3 y-x+2 \sin u-3, \\
f_{2}(x, y, u)=\ln x^{2}+2 x y+y^{2}+2^{x}+\cos u .
\end{gathered}
$$

We define a set-valued mapping $K: \Lambda \rightarrow 2^{X}$ as follows:

$$
K(\lambda):=\{x \in \mathbb{R}:-3+\sin \lambda \leq x \leq 3+\cos \lambda\}, \quad \lambda \in \Lambda .
$$

Then it is easy to see that $1 \in S(0,0)$ and so $S(0,0) \neq$ $\emptyset$. Moreover, it is easy to check that all the assumptions of Theorem 7 are satisfied. Thus, it follows from Theorem 7 that $S$ is u.s.c. at $(0,0)$.

\section{Some Applications}

In this section, we give some applications of Theorem 7 to the optimization problem, the saddle point problem, the Nash equilibria problem, the variational inequality, the variational inequality with set-valued mappings, the equilibrium problem, the generalized strong vector equilibrium problem, and the generalized weak vector equilibrium problem.

Optimization Problem. Let $f: X \rightarrow R$ be a mapping. Let $K$ be a nonempty subset of $X$. A point $x_{0} \in K$ is called a solution of optimization problem if and only if

$$
f\left(x_{0}\right) \leq f(y), \quad \forall y \in K .
$$

Let $f: X \times W \rightarrow R$ be a mapping and let $K: \Lambda \rightarrow 2^{X}$ be a set-valued mapping. For $(u, \lambda) \in W \times \Lambda$, we consider that the following parametric optimization problem consists of finding $x_{0} \in K(\lambda)$ such that

$$
f\left(x_{0}, u\right) \leq f(y, u), \quad \forall y \in K(\lambda) .
$$

Define a solution mapping $O: W \times \Lambda \rightarrow 2^{X}$ to (POP) by

$$
O(u, \lambda)=\{x \in K(\lambda): f(x, u) \leq f(y, u), \forall y \in K(\lambda)\} .
$$

From Theorem 7, we can get the following corollary.

Corollary 10. Let $\left(u_{0}, \lambda_{0}\right) \in W \times \Lambda$. Assume that $K\left(\lambda_{0}\right)$ is nonempty compact, $K(\cdot)$ is continuous at $\lambda_{0}$, and $f(\cdot, \cdot)$ is continuous on $K\left(\lambda_{0}\right) \times\left\{u_{0}\right\}$. Then the mapping $O$ is u.s.c. at $\left(u_{0}, \lambda_{0}\right)$.

Saddle Point Problem. Let $\varphi: X \times X \rightarrow R$ be a mapping. Let $K$ be a nonempty subset of $X$. $\left(\bar{x}_{1}, \bar{x}_{2}\right)$ is called a saddle point on $K$ if and only if

$$
\begin{aligned}
& \left(\bar{x}_{1}, \bar{x}_{2}\right) \in K \times K, \\
& \varphi\left(\bar{x}_{1}, y_{2}\right) \leq \varphi\left(y_{1}, \bar{x}_{2}\right), \quad \forall\left(y_{1}, y_{2}\right) \in K \times K .
\end{aligned}
$$

Let $\varphi: X \times X \times W \rightarrow R$ be a mapping and let $K: \Lambda \rightarrow 2^{X}$ be a set-valued mapping. For $(u, \lambda) \in W \times \Lambda$, we consider that the following parametric saddle point problem consists of finding $\left(\bar{x}_{1}, \bar{x}_{2}\right) \in K(\lambda) \times K(\lambda)$ such that

$$
\varphi\left(\bar{x}_{1}, y_{2}, u\right) \leq \varphi\left(y_{1}, \bar{x}_{2}, u\right), \quad \forall\left(y_{1}, y_{2}\right) \in K(\lambda) \times K(\lambda) .
$$
by

Define a solution mapping $P: W \times \Lambda \rightarrow 2^{X \times X}$ to (PSPP)

$$
\begin{aligned}
P(u, \lambda)=\{ & \left(x_{1}, x_{2}\right) \in K(\lambda) \times K(\lambda): \varphi\left(x_{1}, y_{2}, u\right) \\
& \left.\leq \varphi\left(y_{1}, x_{2}, u\right), \quad \forall\left(y_{1}, y_{2}\right) \in K(\lambda) \times K(\lambda)\right\} .
\end{aligned}
$$

From Theorem 7, we can get the following corollary.

Corollary 11. Let $\left(u_{0}, \lambda_{0}\right) \in W \times \Lambda$. Assume that $K\left(\lambda_{0}\right)$ is nonempty compact, $K(\cdot)$ is continuous at $\lambda_{0}$, and $\varphi(\cdot, \cdot, \cdot)$ is continuous on $K\left(\lambda_{0}\right) \times K\left(\lambda_{0}\right) \times\left\{u_{0}\right\}$. Then the mapping $P$ is u.s.c. at $\left(u_{0}, \lambda_{0}\right)$.

Nash Equilibria Problem. Let $I$ be a finite index set. For every $i \in I$, let $K_{i}$ be a nonempty subset of $X$ and let $f_{i}: \prod_{i \in I} X \rightarrow$ $R$ be a mapping. Let $K:=\prod_{i \in I} K_{i}$. For $x=\left(x_{i}\right)_{i \in I} \in K$, we define $x^{i}=\left(x_{j}\right)_{j \in I, j \neq i}$. A point $\bar{x}=\left(\bar{x}_{i}\right)_{i \in I} \in K$ is called a Nash equilibrium if and only if, for any $i \in I$, we have

$$
f_{i}(\bar{x}) \leq f_{i}\left(\bar{x}^{i}, y_{i}\right), \quad \forall y_{i} \in K_{i} .
$$

For every $i \in I$, let $f_{i}:\left(\prod_{i \in I} X\right) \times W \rightarrow R$ be a mapping and let $K_{i}: \Lambda \rightarrow 2^{X}$ be a set-valued mapping. Let $K(\lambda):=$ $\prod_{i \in I} K_{i}(\lambda)$ for $\lambda \in \Lambda$. For $(u, \lambda) \in W \times \Lambda$, we consider that 
the following parametric Nash equilibria problem consists of finding $\bar{x}=\left(\bar{x}_{i}\right)_{i \in I} \in K(\lambda)$ such that

$$
\forall i \in I, \quad f_{i}(\bar{x}, u) \leq f_{i}\left(\bar{x}^{i}, y_{i}, u\right), \quad \forall y_{i} \in K_{i}(\lambda)
$$

(PNEP)

Define a solution mapping $N: W \times \Lambda \rightarrow 2^{\prod_{i \in I} X}$ to (PNEP) by

$$
\begin{aligned}
N(u, \lambda)=\{ & x=\left(x_{i}\right)_{i \in I} \in K(\lambda): \forall i \in I, \\
& \left.f_{i}(x, u) \leq f_{i}\left(x^{i}, y_{i}, u\right), \forall y_{i} \in K_{i}(\lambda)\right\} .
\end{aligned}
$$

From Theorem 7, we can get the following corollary.

Corollary 12. Let $\left(u_{0}, \lambda_{0}\right) \in W \times \Lambda$. For every $i \in I$, assume that $K_{i}\left(\lambda_{0}\right)$ is nonempty compact, $K_{i}(\cdot)$ is continuous at $\lambda_{0}$, and $f_{i}$ is continuous on $\left(\prod_{i \in I} K_{i}\left(\lambda_{0}\right)\right) \times\left\{u_{0}\right\}$. Then the mapping $N$ is u.s.c. at $\left(u_{0}, \lambda_{0}\right)$.

Variational Inequality. Let $X^{*}$ be the topological dual space of $X$. Let $T: X \rightarrow X^{*}$ be a mapping. Let $K$ be a nonempty subset of $X$. A point $x_{0} \in K$ is called a solution of variational inequality if and only if

$$
\left\langle T\left(x_{0}\right), y-x_{0}\right\rangle \geq 0, \quad \forall y \in K .
$$

Let $T: X \times W \rightarrow X^{*}$ be a mapping and let $K: \Lambda \rightarrow 2^{X}$ be a set-valued mapping. For $(u, \lambda) \in W \times \Lambda$, we consider that the following parametric variational inequality consists of finding $x_{0} \in K(\lambda)$ such that

$$
\left\langle T\left(x_{0}, u\right), y-x_{0}\right\rangle \geq 0, \quad \forall y \in K(\lambda) .
$$

Define a solution mapping $V: W \times \Lambda \rightarrow 2^{X}$ to (PVI) by $V(u, \lambda)=\{x \in K(\lambda):\langle T(x, u), y-x\rangle \geq 0, \forall y \in K(\lambda)\}$.

From Theorem 7, we can get the following corollary.

Corollary 13. Let $\left(u_{0}, \lambda_{0}\right) \in W \times \Lambda$. Assume that $K\left(\lambda_{0}\right)$ is nonempty compact, $K(\cdot)$ is continuous at $\lambda_{0}$, and $T(\cdot, \cdot)$ is continuous on $K\left(\lambda_{0}\right) \times\left\{u_{0}\right\}$. Then the mapping $V$ is u.s.c. at $\left(u_{0}, \lambda_{0}\right)$.

Variational Inequality with Set-Valued Mappings. Let $X^{*}$ be the topological dual space of $X$. Let $T: X \rightarrow 2^{X^{*}}$ be a setvalued mapping. Let $K$ be a nonempty subset of $X$. A point $x_{0} \in K$ is called a solution of variational inequality with setvalued mappings if and only if

$$
\exists \xi \in T\left(x_{0}\right), \quad\left\langle\xi, y-x_{0}\right\rangle \geq 0, \quad \forall y \in K .
$$

Let $T: X \times W \rightarrow 2^{X^{*}}$ and $K: \Lambda \rightarrow 2^{X}$ be two set-valued mappings. For $(u, \lambda) \in W \times \Lambda$, we consider that the following parametric variational inequality with set-valued mappings consists of finding $x_{0} \in K(\lambda)$ such that

$$
\exists \xi \in T\left(x_{0}, u\right), \quad\left\langle\xi, y-x_{0}\right\rangle \geq 0, \quad \forall y \in K(\lambda) .
$$

Define a solution mapping $V_{s}: W \times \Lambda \rightarrow 2^{X}$ to (PVISM) by

$$
\begin{aligned}
V_{s}(u, \lambda)=\{x \in K(\lambda): \exists \xi \in T(x, u), \\
\langle\xi, y-x\rangle \geq 0, \forall y \in K(\lambda)\} .
\end{aligned}
$$

The proof of the following corollary is similar to the proof of Theorem 7. For the convenience of the readers, we also give the proof.

Corollary 14. Let $\left(u_{0}, \lambda_{0}\right) \in W \times \Lambda$. Assume that $K\left(\lambda_{0}\right)$ is nonempty compact, $K(\cdot)$ is continuous at $\lambda_{0}$, and $T(\cdot, \cdot)$ is u.s.c. on $K\left(\lambda_{0}\right) \times\left\{u_{0}\right\}$ with nonempty compact values. Then the mapping $V_{s}$ is u.s.c. at $\left(u_{0}, \lambda_{0}\right)$.

Proof. Suppose to the contrary that $V_{s}(\cdot, \cdot)$ is not u.s.c. at $\left(u_{0}, \lambda_{0}\right)$. Then there exists a neighborhood $W_{0}$ of $V_{s}\left(u_{0}, \lambda_{0}\right)$; for any neighborhood $U\left(u_{0}\right) \times U\left(\lambda_{0}\right)$ of $\left(u_{0}, \lambda_{0}\right)$, there exists $\left(u^{\prime}, \lambda^{\prime}\right) \in U\left(u_{0}\right) \times U\left(\lambda_{0}\right)$ such that $V_{s}\left(u^{\prime}, \lambda^{\prime}\right) \not \subset W_{0}$. Hence, there exists a sequence $\left\{\left(u_{n}, \lambda_{n}\right)\right\}$ with $\left(u_{n}, \lambda_{n}\right) \rightarrow\left(u_{0}, \lambda_{0}\right)$ such that

$$
V_{s}\left(u_{n}, \lambda_{n}\right) \not \subset W_{0}, \quad \forall n \in \mathbb{N} .
$$

Then there exists

$$
x_{n} \in V_{s}\left(u_{n}, \lambda_{n}\right)
$$

such that

$$
x_{n} \notin W_{0}, \quad \forall n \in \mathbb{N} \text {. }
$$

Since $K(\cdot)$ is u.s.c. at $\lambda_{0}$ and $x_{n} \in K\left(\lambda_{n}\right)$, by Lemma 6 , there exist $x_{0} \in K\left(\lambda_{0}\right)$ and a subsequence $\left\{x_{n_{k}}\right\}$ of $\left\{x_{n}\right\}$ such that $x_{n_{k}} \rightarrow x_{0}$. Without loss of generality, we can assume that $x_{n} \rightarrow x_{0}$.

By (24), there exists $\xi_{n} \in T\left(x_{n}, u_{n}\right)$ such that

$$
\left\langle\xi_{n}, y-x_{n}\right\rangle \geq 0, \quad \forall y \in K\left(\lambda_{n}\right) .
$$

Since $\xi_{n} \in T\left(x_{n}, u_{n}\right)$, by Lemma 6 , there exist $\xi_{0} \in T\left(x_{0}, u_{0}\right)$ and a subsequence $\left\{\xi_{n_{k}}\right\}$ of $\left\{\xi_{n}\right\}$ such that $\xi_{n_{k}} \rightarrow \xi_{0}$. Without loss of generality, we can assume that $\xi_{n} \rightarrow \xi_{0}$.

For any $\bar{y} \in K\left(\lambda_{0}\right)$, by Lemma 5, there exists $y_{n} \in K\left(\lambda_{n}\right)$ such that $y_{n} \rightarrow \bar{y}$. Noting (26), we have

$$
\left\langle\xi_{n}, y_{n}-x_{n}\right\rangle \geq 0 \text {. }
$$

It follows from $\left\langle\xi_{n}, y_{n}-x_{n}\right\rangle \rightarrow\left\langle\xi_{0}, \bar{y}-x_{0}\right\rangle$ that

$$
\left\langle\xi_{0}, \bar{y}-x_{0}\right\rangle \geq 0 \text {. }
$$

Therefore, $x_{0} \in V_{s}\left(u_{0}, \lambda_{0}\right)$. We can see that $x_{n} \rightarrow x_{0} \in W_{0}$, which contradicts with (25).

Equilibrium Problem. Let $f: X \times X \rightarrow R$ be a mapping. Let $K$ be a nonempty subset of $X$. A point $x_{0} \in K$ is called a solution of equilibrium problem if and only if

$$
f\left(x_{0}, y\right) \geq 0, \quad \forall y \in K .
$$


Let $f: X \times X \times W \rightarrow R$ and $K: \Lambda \rightarrow 2^{X}$ be two setvalued mappings. For $(u, \lambda) \in W \times \Lambda$, we consider that the following parametric equilibrium problem consists of finding $x_{0} \in K(\lambda)$ such that

$$
f\left(x_{0}, y, u\right) \geq 0, \quad \forall y \in K(\lambda) .
$$

Define a solution mapping $E: W \times \Lambda \rightarrow 2^{X}$ to (PEP) by

$$
E(u, \lambda)=\{x \in K(\lambda): f(x, y, u) \geq 0, \forall y \in K(\lambda)\} .
$$

From Theorem 7, we can get the following corollary.

Corollary 15. Let $\left(u_{0}, \lambda_{0}\right) \in W \times \Lambda$. Assume that $K\left(\lambda_{0}\right)$ is nonempty compact, $K(\cdot)$ is continuous at $\lambda_{0}$, and $f(\cdot, \cdot, \cdot)$ is continuous on $K\left(\lambda_{0}\right) \times K\left(\lambda_{0}\right) \times\left\{u_{0}\right\}$. Then the mapping $E$ is u.s.c. at $\left(u_{0}, \lambda_{0}\right)$.

Generalized Strong Vector Equilibrium Problem. Assume that $C \subseteq Y$ is a closed cone. Let $F: X \times X \rightarrow 2^{Y}$ be a set-valued mapping. Let $K$ be a nonempty subset of $X$. A point $x_{0} \in K$ is called a solution of generalized strong vector equilibrium problem if and only if

$$
F\left(x_{0}, y\right) \subseteq C, \quad \forall y \in K .
$$

Let $F: X \times X \times W \rightarrow 2^{Y}$ and $K: \Lambda \rightarrow 2^{X}$ be two setvalued mappings. For $(u, \lambda) \in W \times \Lambda$, we consider that the following parametric generalized strong vector equilibrium problem consists of finding $x_{0} \in K(\lambda)$ such that

$$
F\left(x_{0}, y, u\right) \subseteq C, \quad \forall y \in K(\lambda) .
$$

(PGSVEP)

Define a solution mapping $M: W \times \Lambda \rightarrow 2^{X}$ to (PGSVEP) by

$$
M(u, \lambda)=\{x \in K(\lambda): F(x, y, u) \subseteq C, \forall y \in K(\lambda)\} .
$$

From Theorem 7, we can get the following corollary.

Corollary 16. Let $\left(u_{0}, \lambda_{0}\right) \in W \times \Lambda$. Assume that $K\left(\lambda_{0}\right)$ is nonempty compact, $K(\cdot)$ is continuous at $\lambda_{0}$, and $F(\cdot, \cdot, \cdot)$ is l.s.c. on $K\left(\lambda_{0}\right) \times K\left(\lambda_{0}\right) \times\left\{u_{0}\right\}$. Then the mapping $M$ is u.s.c. at $\left(u_{0}, \lambda_{0}\right)$.

Generalized Weak Vector Equilibrium Problem. Assume that $C \subseteq Y$ is a cone with nonempty interior. Let $F: X \times X \rightarrow 2^{Y}$ be a set-valued mapping. Let $K$ be a nonempty subset of $X$. A point $x_{0} \in K$ is called a solution of generalized weak vector equilibrium problem if and only if

$$
F\left(x_{0}, y\right) \cap(-\operatorname{int} C)=\emptyset, \quad \forall y \in K .
$$

Let $F: X \times X \times W \rightarrow 2^{Y}$ and $K: \Lambda \rightarrow 2^{X}$ be two setvalued mappings. For $(u, \lambda) \in W \times \Lambda$, we consider that the following parametric generalized weak vector equilibrium problem consists of finding $x_{0} \in K(\lambda)$ such that

$$
F\left(x_{0}, y, u\right) \cap(-\operatorname{int} C)=\emptyset, \quad \forall y \in K(\lambda) . \quad \text { (PGWVEP) }
$$

Define a solution mapping $G: W \times \Lambda \rightarrow 2^{X}$ to (PGWVEP) by

$$
\begin{aligned}
& G(u, \lambda) \\
& \quad=\{x \in K(\lambda): F(x, y, u) \cap(-\operatorname{int} C)=\emptyset, \forall y \in K(\lambda)\} .
\end{aligned}
$$

From Theorem 7, we can get the following corollary.

Corollary 17. Let $\left(u_{0}, \lambda_{0}\right) \in W \times \Lambda$. Assume that $K\left(\lambda_{0}\right)$ is nonempty compact, $K(\cdot)$ is continuous at $\lambda_{0}$, and $F(\cdot, \cdot, \cdot)$ is l.s.c. on $K\left(\lambda_{0}\right) \times K\left(\lambda_{0}\right) \times\left\{u_{0}\right\}$. Then the mapping $G$ is u.s.c. at $\left(u_{0}, \lambda_{0}\right)$.

Remark 18. Corollary 17 is similar to Theorem 3.4 of [18].

From Corollary 17, it is easy to get the following corollary.

Corollary 19. Let $\left(u_{0}, \lambda_{0}\right) \in W \times \Lambda$. Assume that $K\left(\lambda_{0}\right)$ is nonempty compact, $K(\cdot)$ is continuous at $\lambda_{0}$, and $F(\cdot, \cdot, \cdot)$ is $C$ l.s.c. on $K\left(\lambda_{0}\right) \times K\left(\lambda_{0}\right) \times\left\{u_{0}\right\}$. Then $G$ is u.s.c. at $\left(u_{0}, \lambda_{0}\right)$.

Remark 20. In the proof of upper semicontinuity of solution mapping, Corollary 19 improves Theorem 3.1 of [15].

\section{Conflict of Interests}

The authors declare that there is no conflict of interests regarding the publication of this paper.

\section{Acknowledgments}

The authors are grateful to the editor and the referees for their valuable comments and suggestions. This work was supported by the National Natural Science Foundation of China (11171237, 11471230).

\section{References}

[1] G. Y. Chen, X. X. Huang, and X. Q. Yang, Vector Optimization: Set-valued and Variational Analysis, vol. 541 of Lecture Notes in Economics and Mathematical Systems, Springer, Berlin, Germany, 2005.

[2] F. Giannessi, Ed., Vector Variational Inequalities and Vector Equilibria, Kluwer Academic Publishers, Dordrecht, The Netherlands, 2000.

[3] G. Y. Chen, "Existence of solutions for a vector variational inequality: an extension of the Hartmann-Stampacchia theorem," Journal of Optimization Theory and Applications, vol. 74, no. 3, pp. 445-456, 1992.

[4] Q. H. Ansari, I. V. Konnov, and J. C. Yao, "Existence of a solution and variational principles for vector equilibrium problems," Journal of Optimization Theory and Applications, vol. 110, no. 3, pp. 481-492, 2001.

[5] Q. H. Ansari, "Existence of solutions of systems of generalized implicit vector quasi-equilibrium problems," Journal of Mathematical Analysis and Applications, vol. 341, no. 2, pp. 1271-1283, 2008.

[6] X. H. Gong, "Strong vector equilibrium problems," Journal of Global Optimization, vol. 36, no. 3, pp. 339-349, 2006. 
[7] N.-J. Huang and Y.-P. Fang, "On vector variational inequalities in reflexive Banach spaces," Journal of Global Optimization, vol. 32, no. 4, pp. 495-505, 2005.

[8] N. J. Huang, J. Li, and J. C. Yao, "Gap functions and existence of solutions for a system of vector equilibrium problems," Journal of Optimization Theory and Applications, vol. 133, no. 2, pp. 201212, 2007.

[9] L. Q. Anh and P. Q. Khanh, "On the stability of the solution sets of general multivalued vector quasiequilibrium problems," Journal of Optimization Theory and Applications, vol. 135, no. 2, pp. 271-284, 2007.

[10] L. Q. Anh and P. Q. Khanh, "Continuity of solution maps of parametric quasiequilibrium problems," Journal of Global Optimization, vol. 46, no. 2, pp. 247-259, 2010.

[11] N. Dinh, M. A. Goberna, and M. A. López, "On the stability of the optimal value and the optimal set in optimization problems," Journal of Convex Analysis, vol. 19, no. 4, pp. 927-953, 2012.

[12] N. J. Huang, J. Li, and H. B. Thompson, "Stability for parametric implicit vector equilibrium problems," Mathematical and Computer Modelling, vol. 43, no. 11-12, pp. 1267-1274, 2006.

[13] X. H. Gong, "Continuity of the solution set to parametric weak vector equilibrium problems," Journal of Optimization Theory and Applications, vol. 139, no. 1, pp. 35-46, 2008.

[14] C. R. Chen, S. J. Li, and K. L. Teo, "Solution semicontinuity of parametric generalized vector equilibrium problems," Journal of Global Optimization, vol. 45, no. 2, pp. 309-318, 2009.

[15] B. Chen and N. J. Huang, "Continuity of the solution mapping to parametric generalized vector equilibrium problems," Journal of Global Optimization, vol. 56, no. 4, pp. 1515-1528, 2013.

[16] R. Lucchetti and F. Patrone, "Closure and upper semicontinuity results in mathematical programming, Nash and economic equilibria," Optimization, vol. 17, no. 5, pp. 619-628, 1986.

[17] T. Tanino and Y. Sawaragi, "Stability of nondominated solutions in multicriteria decision-making," Journal of Optimization Theory and Applications, vol. 30, no. 2, pp. 229-253, 1980.

[18] L. Q. Anh and P. Q. Khanh, "Semicontinuity of the solution set of parametric multivalued vector quasiequilibrium problems," Journal of Mathematical Analysis and Applications, vol. 294, no. 2, pp. 699-711, 2004.

[19] P. Q. Khanh and L. M. Luu, "Upper semicontinuity of the solution set to parametric vector quasivariational inequalities," Journal of Global Optimization, vol. 32, no. 4, pp. 569-580, 2005.

[20] Y.-P. Fang and N.-J. Huang, "Upper semicontinuity of the solution maps in homogeneous vector quasi-equilibrium problems," Optimization, vol. 55, no. 3, pp. 231-239, 2006.

[21] Y. H. Cheng and D. L. Zhu, "Global stability results for the weak vector variational inequality," Journal of Global Optimization, vol. 32, no. 4, pp. 543-550, 2005.

[22] S. J. Li and Z. M. Fang, "Lower semicontinuity of the solution mappings to a parametric generalized Ky Fan inequality," Journal of Optimization Theory and Applications, vol. 147, no. 3, pp. 507-515, 2010.

[23] X. H. Gong and J. C. Yao, "Lower semicontinuity of the set of efficient solutions for generalized systems," Journal of Optimization Theory and Applications, vol. 138, no. 2, pp. 197205, 2008.

[24] S. J. Li, H. M. Liu, Y. Zhang, and Z. M. Fang, "Continuity of the solution mappings to parametric generalized strong vector equilibrium problems," Journal of Global Optimization, vol. 55, no. 3, pp. 597-610, 2013.
[25] W. Y. Zhang, Z. M. Fang, and Y. Zhang, "A note on the lower semicontinuity of efficient solutions for parametric vector equilibrium problems," Applied Mathematics Letters, vol. 26, no. 4, pp. 469-472, 2013.

[26] X. D. Fan, C. Z. Cheng, and H. J. Wang, "Stability analysis for vector quasiequilibrium problems," Positivity, vol. 17, no. 3, pp. 365-379, 2013.

[27] Y. D. Xu and S. J. Li, "On the lower semicontinuity of the solution mappings to a parametric generalized strong vector equilibrium problem," Positivity, vol. 17, no. 2, pp. 341-353, 2013.

[28] Y. Han and X. H. Gong, "Lower semicontinuity of solution mapping to parametric generalized strong vector equilibrium problems," Applied Mathematics Letters, vol. 28, pp. 38-41, 2014.

[29] J.-P. Aubin and I. Ekeland, Applied Nonlinear Analysis, John Wiley \& Sons, New York, NY, USA, 1984.

[30] A. Göpfert, H. Riahi, C. Tammer, and C. Zălinescu, Variational methods in partially ordered spaces, Springer, New York, NY, USA, 2003. 


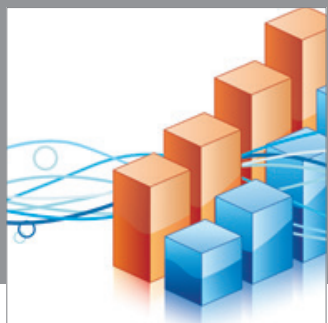

Advances in

Operations Research

mansans

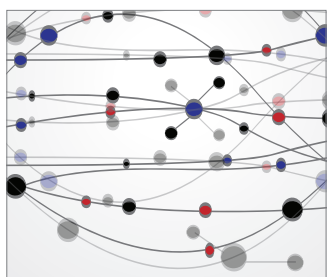

The Scientific World Journal

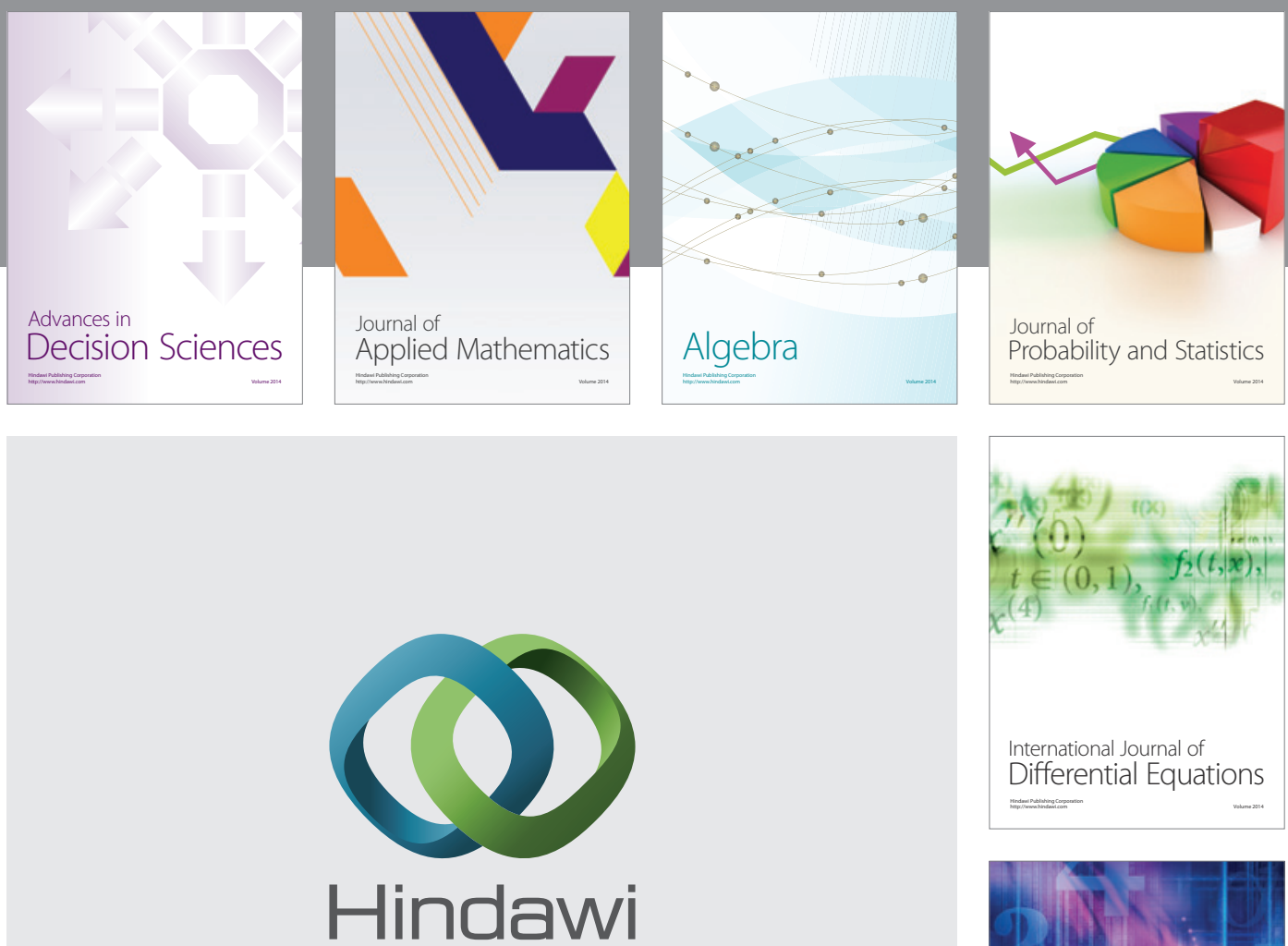

Submit your manuscripts at http://www.hindawi.com
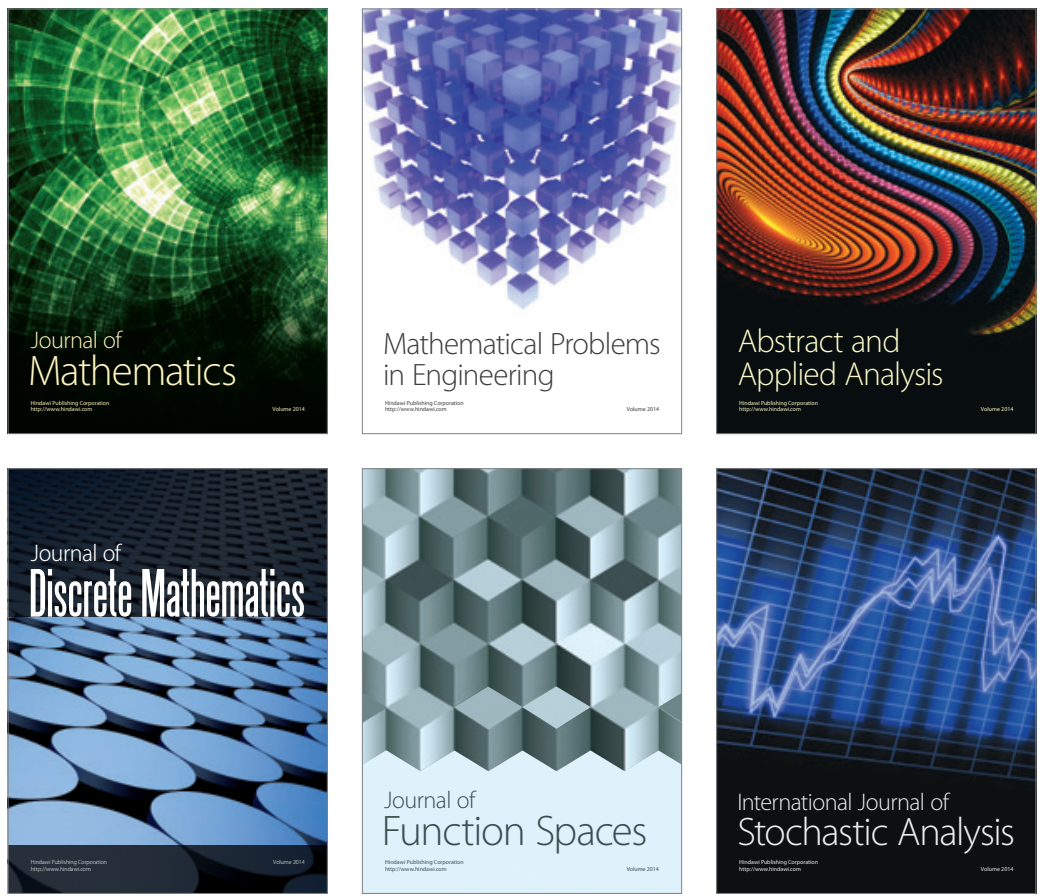

Journal of

Function Spaces

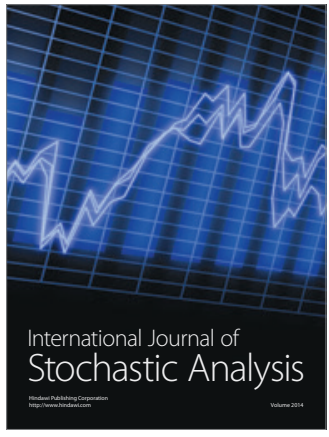


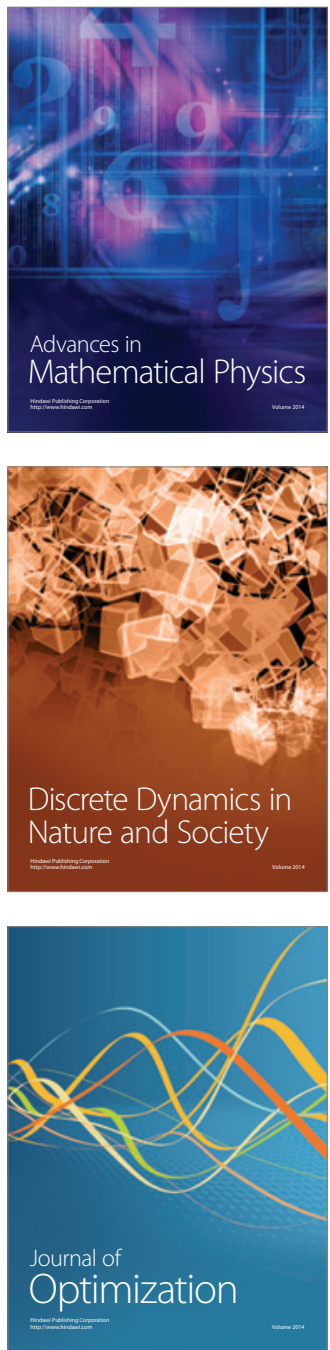